\title{
Humboldt's spa: microbial diversity is controlled by temperature in geothermal environments
}

\author{
Christine E Sharp ${ }^{1,5}$, Allyson L Brady ${ }^{1,5}$, Glen H Sharp ${ }^{2}$, Stephen E Grasby ${ }^{3}$, \\ Matthew B Stott ${ }^{4}$ and Peter F Dunfield ${ }^{1}$ \\ ${ }^{1}$ Department of Biological Sciences, University of Calgary, Calgary, Alberta, Canada; ${ }^{2}$ Niagara Catholic \\ District School Board, Welland, Ontario, Canada; ${ }^{3}$ Geological Survey of Canada, Calgary, Alberta, \\ Canada and ${ }^{4}$ GNS Science, Extremophile Research Group, Taupo, New Zealand
}

\begin{abstract}
Over 200 years ago Alexander von Humboldt (1808) observed that plant and animal diversity peaks at tropical latitudes and decreases toward the poles, a trend he attributed to more favorable temperatures in the tropics. Studies to date suggest that this temperature-diversity gradient is weak or nonexistent for Bacteria and Archaea. To test the impacts of temperature as well as $\mathrm{pH}$ on bacterial and archaeal diversity, we performed pyrotag sequencing of 16S rRNA genes retrieved from 165 soil, sediment and biomat samples of 36 geothermal areas in Canada and New Zealand, covering a temperature range of $7.5-99^{\circ} \mathrm{C}$ and a pH range of $1.8-9.0$. This represents the widest ranges of temperature and $\mathrm{pH}$ yet examined in a single microbial diversity study. Species richness and diversity indices were strongly correlated to temperature, with $R^{2}$ values up to 0.62 for neutralalkaline springs. The distributions were unimodal, with peak diversity at $24{ }^{\circ} \mathrm{C}$ and decreasing diversity at higher and lower temperature extremes. There was also a significant pH effect on diversity; however, in contrast to previous studies of soil microbial diversity, pH explained less of the variability (13-20\%) than temperature in the geothermal samples. No correlation was observed between diversity values and latitude from the equator, and we therefore infer a direct temperature effect in our data set. These results demonstrate that temperature exerts a strong control on microbial diversity when considered over most of the temperature range within which life is possible.
\end{abstract}

The ISME Journal (2014) 8, 1166-1174; doi:10.1038/ismej.2013.237; published online 16 January 2014

Subject Category: Microbial population and community ecology

Keywords: diversity; hot spring; extremphile; thermophile; pyrotag sequencing

\section{Introduction}

A fundamental goal of ecology is to understand how living communities are organized in response to environmental parameters. One general trend is the increasing diversity of macroorganisms such as plants and animals toward mid-latitudes, first described by Forster (1778) and Humboldt (1808), and supported by many subsequent investigations (for examples see: Gaston, 2000; Allen et al., 2002; Hawkins et al., 2003). Humboldt attributed the pattern to the intuitively simple idea that tropical climates are optimal for life and fewer species can physiologically tolerate more extreme conditions. This concept is now known under several guises such as the 'metabolic niche hypothesis' (Clarke and Gaston, 2006), the 'physiological

Correspondence: PF Dunfield, Department of Biological Sciences, University of Calgary, 2500 University Dr. NW, Calgary, Alberta T2N 1N4, Canada.

E-mail: pfdunfie@ucalgary.ca

${ }^{5}$ These authors contributed equally to this work

Received 15 May 2013; revised 7 November 2013; accepted 9 December 2013; published online 16 January 2014 tolerance hypothesis' (Currie et al., 2004) or the 'range-limitation hypothesis' (Evans et al., 2005), which all propose that fewer species are adapted to cold arctic conditions compared with warm tropical conditions (Clarke and Gaston, 2006). However, the observed latitude-diversity pattern has also been attributed to other causes including increased climate stability and higher speciation rates, habitat area, population sizes and net primary productivity (NPP) in the tropics (Gaston, 2000; Clarke and Gaston, 2006). Although many of these factors are influenced by temperature, whether temperature itself has any direct influence on diversity is still a matter of debate.

Only recently have high-throughput tools (for example, $16 \mathrm{~S}$ rRNA gene pyrotag sequencing) become available to reliably examine patterns of microbial diversity. It is generally assumed that environmental parameters such as salinity, temperature, water availability and $\mathrm{pH}$ influence the composition of microbial communities, with decreasing diversity as conditions become more extreme (Oren, 2001; Fierer and Jackson, 2006). However, with the exception of $\mathrm{pH}$, strong data sets to support this 
assumption are rare, and the relative importance of different environmental factors remains unresolved. Remarkably, there is little evidence for a latitudediversity or temperature-diversity gradient in Bacteria and Archaea. Two studies noted weak relationships of phytoplankton diversity to latitude and temperature in oceanic samples, with $R^{2}$ values of 0.1-0.2 (Pommier et al., 2007; Fuhrman et al., 2008). However, large-scale investigations of soil have attributed changes in microbial diversity predominantly to variations in $\mathrm{pH}$, with almost none of the variability explained by temperature (Pennanen et al., 1999; Fierer and Jackson, 2006; Chu et al., 2010; Rousk et al., 2010). Studies have noted a clear peak of diversity at neutral $\mathrm{pH}$ when comparing soils across varying spatial scales and soil types (Fierer and Jackson, 2006), including chalk-treated (Rousk et al., 2010) and arctic soils (Chu et al., 2010). In these studies temperature explained little of the overall variability in diversity. However, the temperatures were all within a limited range, typically estimated as mean annual temperatures from -10 to $30^{\circ} \mathrm{C}$. As some microbes are capable of growth up to $122^{\circ} \mathrm{C}$ (Takai et al., 2008), investigations of microbial diversity across much larger temperature ranges are warranted.

Examining microbial communities in geothermal systems has several advantages for testing a temperature-diversity effect compared with examining latitudinal gradients: (i) the range of potential temperatures is much higher; (ii) temperatures are relatively stable and therefore communities should fluctuate little seasonally; and (iii) temperatures are controlled by geothermal processes and therefore uncoupled from other latitudinal gradients such as NPP. However, studies comparing diversity across geothermal systems remain few in number and consistent trends remain unresolved. Although some studies have noted a lack of correlation (Yim et al., 2006; Huang et al., 2011; Hou et al., 2013; Wang et al., 2013) or even a slight increase in diversity with increasing temperature (Purcell et al., 2007), others have noted a decrease in diversity with increasing temperature (Miller et al., 2009; Tobler and Benning, 2011; Everroad et al., 2012; Cole et al., 2013). Although these studies hint at underlying general patterns, they are all limited by at least one of several shortcomings: restricted methodologies that detect only the few most abundant operational taxonomic units (OTUs), such as construction of clone libraries (Yim et al., 2006; Purcell et al., 2007; Miller et al., 2009; Huang et al., 2011; Tobler and Benning, 2011; Everroad et al., 2012); low numbers of samples $(<10)$ (Yim et al., 2006; Purcell et al., 2007; Miller et al., 2009; Tobler and Benning, 2011; Everroad et al., 2012); samples taken from a single geothermal field and therefore possibly showing site-specific trends (Yim et al., 2006; Miller et al., 2009; Everroad et al., 2012; Cole et al., 2013); or a limited temperature range $\left(<30^{\circ} \mathrm{C}\right.$ total range) (Purcell et al., 2007; Miller et al., 2009; Everroad et al., 2012; Cole et al., 2013).
Two recent studies based on 16S rRNA gene pyrotag sequencing, the technique that assesses microbial diversity to the most depth, support the notion that extreme temperatures may restrict microbial diversity (Everroad et al., 2012; Cole et al., 2013), but other pyrotag sequencing studies with a larger number of samples and a broader temperature range do not (Hou et al., 2013; Wang et al., 2013). The relative importance of $\mathrm{pH}$ versus temperature is also unknown as few studies cover wide-ranges of both temperature and $\mathrm{pH}$.

The objective of this study was to test for a temperature-diversity gradient in geothermal environments covering a much broader temperature range using 16S rRNA gene pyrotag sequencing. We collected 165 geochemically diverse samples from Canada and New Zealand. To the best of our knowledge, this represents the largest sample set (number of sites and sequences) and the broadest range of both temperature and $\mathrm{pH}$ yet examined. Our aims were: (i) to evaluate the relative influences of temperature and $\mathrm{pH}$ on microbial diversity and (ii) to test whether temperature has a direct effect on diversity in ecosystems where there are no confounding aspects of latitude. Examining microbial communities in hot springs has a key advantage for testing the temperature-diversity effect compared with examining macro or microflora over latitudinal gradients covering thousands of kilometers; the temperatures are controlled by geothermal processes and are therefore uncoupled from factors such as latitude, NPP and the magnitude of seasonal variability.

\section{Materials and methods}

\section{Geothermal spring sample collection}

Study sites were selected from springs in Western Canada (Grasby et al., 2000) and the Taupo Volcanic Zone, New Zealand (Giggenbach, 1994). As it is generally accepted that planktonic communities are less diverse than soil, sediment or biomat communities, we sampled only the latter. One hundred and sixty-five sediment, soil and mat samples were collected from 36 distinct geothermal sites. Samples were collected at various times of the year between 2009 and 2012, representing a temperature range of 7.5-99 ${ }^{\circ} \mathrm{C}$ and a $\mathrm{pH}$ range of 1.8-9.0 (Supplementary Table S1). Samples were immediately placed at $4{ }^{\circ} \mathrm{C}$ and frozen at $-80^{\circ} \mathrm{C}$ within 2 days of collection.

\section{Microbial community analysis}

DNA was extracted from $0.5 \mathrm{~g}(+/-5 \mathrm{mg})$ of each soil using the FastDNA Extraction Kit for Soil (MP Biomedicals, Santa Ana, CA, USA), with additional purification steps using $5.5 \mathrm{M}$ guanidine thiocyanate (Knief et al., 2003). DNA concentration was determined with a Qubit Fluorometer using a Quant-iT 
dsDNA HS Assay Kit (Invitrogen, Carlsbad, CA, USA).

16S rRNA genes were amplified from the DNA extracts using FLX Titanium amplicon primers 454T_RA_X and 454T_F, which contain 16S rRNA gene targeted primers $926 \mathrm{fw}$ ( $5^{\prime}$-aaactYaaaKgaattgRcgg- $\left.3^{\prime}\right)$ and 1392r ( $5^{\prime}$-acgggcggtgtgtRc- $\left.3^{\prime}\right)$ at their $3^{\prime}$ ends and adapters necessary for the Roche Titanium chemistry (Ramos-Padrón et al., 2011). Universal primers 926fw and 1392r encompass the V8 variable region of the $16 \mathrm{~S}$ rRNA gene from Bacteria and Archaea as well as the 18S rRNA gene in Eukarya (Engelbrektson et al., 2010). Each reverse primer contains a unique 10-nucleotide multiplex identifier barcode sequence that allows sequences to be binned according to sample.

PCR reaction mixtures contained $0.04 \mu \mathrm{M}$ of the forward primer, $25 \mu \mathrm{l}$ of $2 \times$ Premix F (Interscience, Saint Nom, France), 1.25 U Taq DNA polymerase (Fermentas, Fisher Scientific, Ottawa, ON, Canada), $0.04 \mu \mathrm{M}$ of the reverse primer (with its unique barcode sequence) and $2 \mu \mathrm{l}$ of template DNA, made up to $50 \mu \mathrm{l}$ total with nuclease-free water (Qiagen, Toronto, ON, Canada). PCR reaction conditions were: initial denaturation at $95{ }^{\circ} \mathrm{C}$ for $3 \mathrm{~min}$, followed by 35 cycles of $30 \mathrm{~s}$ at $95^{\circ} \mathrm{C}, 45 \mathrm{~s}$ at $55^{\circ} \mathrm{C}$ and $90 \mathrm{~s}$ at $72^{\circ} \mathrm{C}$, and a 10 min final elongation at $72^{\circ} \mathrm{C}$. PCR products were visualized on a $1 \%$ agarose gel and purified with an EZ-10 Spin Column PCR Purification Kit (BioBasic Inc., Markham, ON, Canada). DNA concentration was determined with a Qubit Fluorometer using a QuantiT dsDNA HS Assay Kit (Invitrogen). Purified PCR products ( $\sim 150 \mathrm{ng}$ total DNA) were analyzed at the Genome Quebec and McGill University Innovation Centre, Montreal, Quebec on a 454 Life Sciences Genome Sequencer FLX (Roche, Branford, CT, USA) machine running the Titanium chemistry.

\section{Processing of pyrosequencing data}

Raw sequence data were analyzed using the Quantitative Insights Into Microbial Ecology (QIIME) pipeline version 1.3 (Caporaso et al., 2010a). Briefly, sequences shorter than $375 \mathrm{bp}$ or longer than $600 \mathrm{bp}$ and with a quality score lower than 25 were eliminated, and the $10 \mathrm{bp}$ barcode was used to assign sequences to samples. Forward primers, reverse primers and the barcode were removed from the final sequences. OTUs were identified using uclust (Edgar, 2010) with thresholds of 97\%, 95\% and $90 \%$ sequence similarity. A representative sequence for each OTU (the most abundant sequence within the OTU) was selected and screened using ChimeraSlayer (Haas et al., 2011). Identified chimeric sequences were removed from downstream analysis. Representative sequences were aligned using PyNAST (Caporaso et al., 2010b) and a phylogenetic tree built using FastTree (Price et al., 2009). Taxonomic identification of each OTU was determined using nucleotide BLAST (Altschul et al., 1990) against the Silva 108 core set (Pruesse et al., 2007). Eukaryotic (including chloroplast) sequences were removed from further analysis. The final high quality pyrotag sequencing data set yielded a total of 1162553 sequences.

Alpha diversity metrics, including Shannon diversity index, Chao1 (Chao, 1984), Faith's phylogenetic diversity (Faith, 1992) and the number of observed OTUs per sample were calculated using QIIME by rarifying all samples to the same sequencing depth (2730 sequences per sample), resulting in the exclusion of five sites. Above a sequencing depth of 700 there was little increase in the Shannon diversity index with further sampling (data not shown). Correlations between the diversity estimates and the $\mathrm{pH}$ and temperature were tested for significance using SYSTAT 13.0. Best-fit modeling of Shannon, Chao1, Faith's phylogenetic diversity and number of observed OTUs per sample were performed in SigmaPlot v 12.3 using a linear regression and a four-parameter Gaussian curve fit function. To confirm the observed trends, multiple quality-filtering steps were performed, including denoising of the entire data set using the built-in QIIME denoiser workflow (at default settings), removal of OTUs that were only present once in the data set and removal of OTUs that only appeared in one sample in the data set. Pyrotag sequencing has an error rate of $\sim 1 \%$ (Gilles et al., 2011), however this was not expected to influence any trends in diversity as all samples would be equally affected.

Differences in the overall community composition between samples were determined using the BrayCurtis and UniFrac distance metrics. Pairwise distances calculated for the total community analyses were visualized using nonmetric multidimensional scaling plots as implemented in QIIME.

Nucleotide sequence accession numbers Pyrotag read data sets have been submitted to the SRA under accession number SRP028305.

\section{Results}

Analysis of the 454-pyrotag sequencing data set A total of 1162553 high quality sequences were obtained from 165 samples with 634-15 283 sequences per sample (mean 7045), and an average sequence length of $451 \mathrm{bp}$. When sequences were clustered at the $97 \%$ similarity level, there were 61910 OTUs in the data set. Grouping at 95\% similarity resulted in 37406 OTUs, whereas grouping at $90 \%$ similarity resulted in 14769 OTUs.

\section{Alpha diversity versus temperature and $p H$}

Multiple linear regression was performed for alpha diversity indices versus temperature and $\mathrm{pH}$. Combined, the two factors accounted for about half the 
variability in diversity, with $R^{2}=0.50$ for the Shannon index; 0.56 for total OTUs detected (log transformed for normality); 0.49 for Faith's phylogenetic distance (log transformed) and 0.56 for Chao1 (log transformed). Temperature had a greater relationship to these indices than did $\mathrm{pH}$, with linear regression $R^{2}$ values of $0.31-0.36$ for temperature alone (Supplementary Figure S1). A Gaussian curve fit increased the goodness-of-fit of the relationships to temperature up to $R^{2}=0.40-0.45$ (Supplementary Figure S1). Similar temperature effects were observed when samples were clustered into OTUs at 95\% and 90\% identity (Supplementary Figure S2), indicating that the data are robust at different species definitions, and not sensitive to sequencing errors.

To confirm the robustness of the observed trends to different data filtering methods we performed several additional quality control steps. OTUs that were only present once in the data set and OTUs that only appeared in one sample in the data set were removed (Supplementary Figure S3). Alternatively, we denoised the entire data set using the QIIME denoiser to ensure the observed trends were not a result of pyrosequencing errors (Supplementary Figure S4). Each of these three filtering steps caused the total number of OTUs in our data set to decrease by $50-60 \%$, however on a per sample basis they caused only a slight decline in diversity, as the removed OTUs were generally limited to a single sample (Supplementary Table S2). Shannon index was particularly insensitive to the filtering because rare OTUs contribute less to the index total (Supplementary Table S2). Most importantly, the fundamental nature of the relationships between alpha diversity indices and temperature were not affected by any of the three filtering methods (Supplementary Figures S3 and S4).

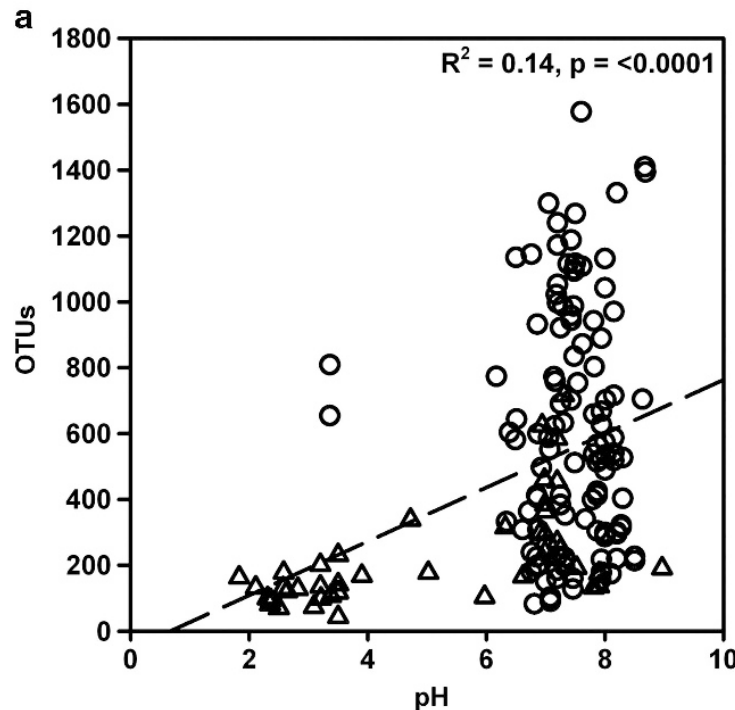

Hot springs generally fall into two categories, acid springs versus neutral to slightly alkaline springs, as evident by the two clouds of points on the diversity versus $\mathrm{pH}$ plots (Figure 1, Supplementary Figure S1). The neutral-alkaline springs had consistently higher alpha diversity than the acid springs, and linear regression $R^{2}$ values of all diversity indices versus $\mathrm{pH}$ were between $0.13-0.20$ (Figure 1, Supplementary Figure S1). Nonmetric multidimensional scaling plots also showed that acid spring communities separated from those in neutral and alkaline springs regardless of temperature (Figure 2, Supplementary Figure S7). Therefore, to best represent the temperature trend without confounding effects of $\mathrm{pH}$, species richness (OTUs) and Shannon diversity were plotted against temperature for neutral-alkaline springs only (Figure 3). Species richness and the Shannon index each showed strong relationships to temperature for the neutral-alkaline ( $\mathrm{pH}>5$ ) springs from this study, with high $R^{2}$ values of 0.51 and peak species richness near $24^{\circ} \mathrm{C}$ (Figure 3).

To further verify the temperature-diversity relationship, Shannon diversity index data from two recent pyrotag sequencing studies of neutralalkaline hot springs in China and the US (Cole et al., 2013; Hou et al., 2013) were included in Figure 3b. To conform with our sample set, only sediment and biomat samples were included, whereas water samples were ignored. We included only Shannon index data because it is less sensitive than OTU richness to different sampling depths and filtering methods (Supplementary Table S2). Although the analysis pipelines were not identical among the different studies, they were similar. Primers used by Cole et al. (2013) are the same primers used in the current study and amplified the V6-V8 region of the 16S rRNA gene. Primers from

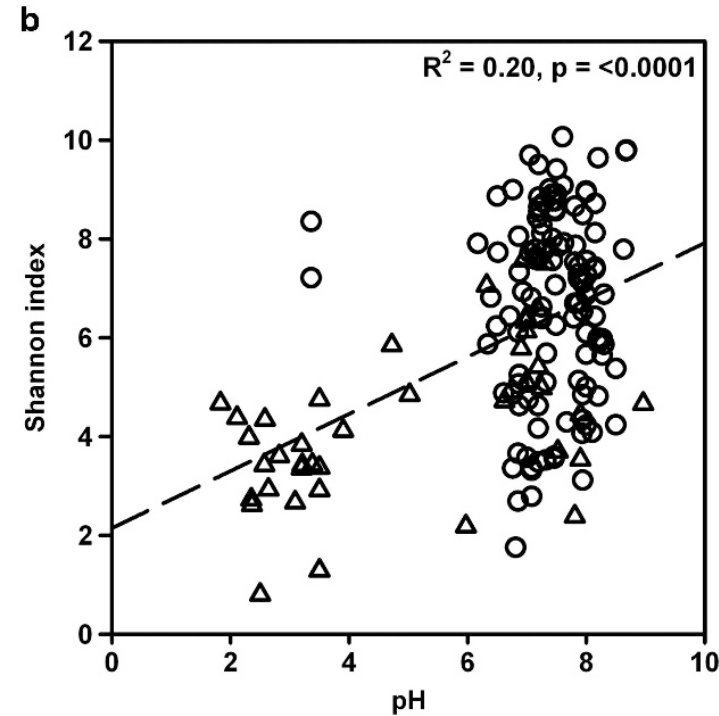

Figure 1 Scatter plots of total OTUs detected (a) and Shannon diversity index (b) versus sample pH. Dashed lines represent the best-fit linear model to the data. Diversity indices were calculated using 2730 sequences per spring sample. Symbol shape indicates Canada $(O$ ) or New Zealand $(\Delta)$. 

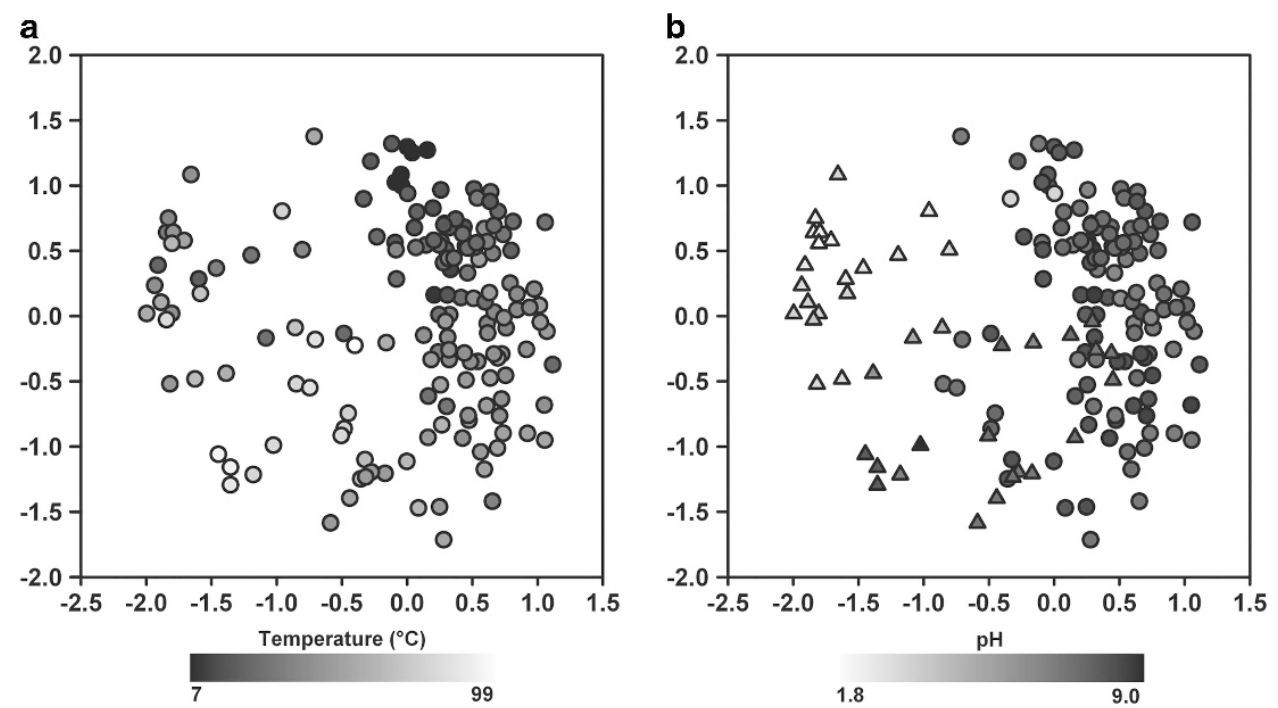

Figure 2 Nonmetric multidimensional scaling plot of geothermal spring microbial communities based on Bray-Curtis similarity showing separation of sites based on temperature and $\mathrm{pH}$. Shade coding indicates temperature (a) or $\mathrm{pH}(\mathbf{b})$. Symbols in (b) indicate location: Canada $(\bigcirc)$ or New Zealand $(\Delta)$.
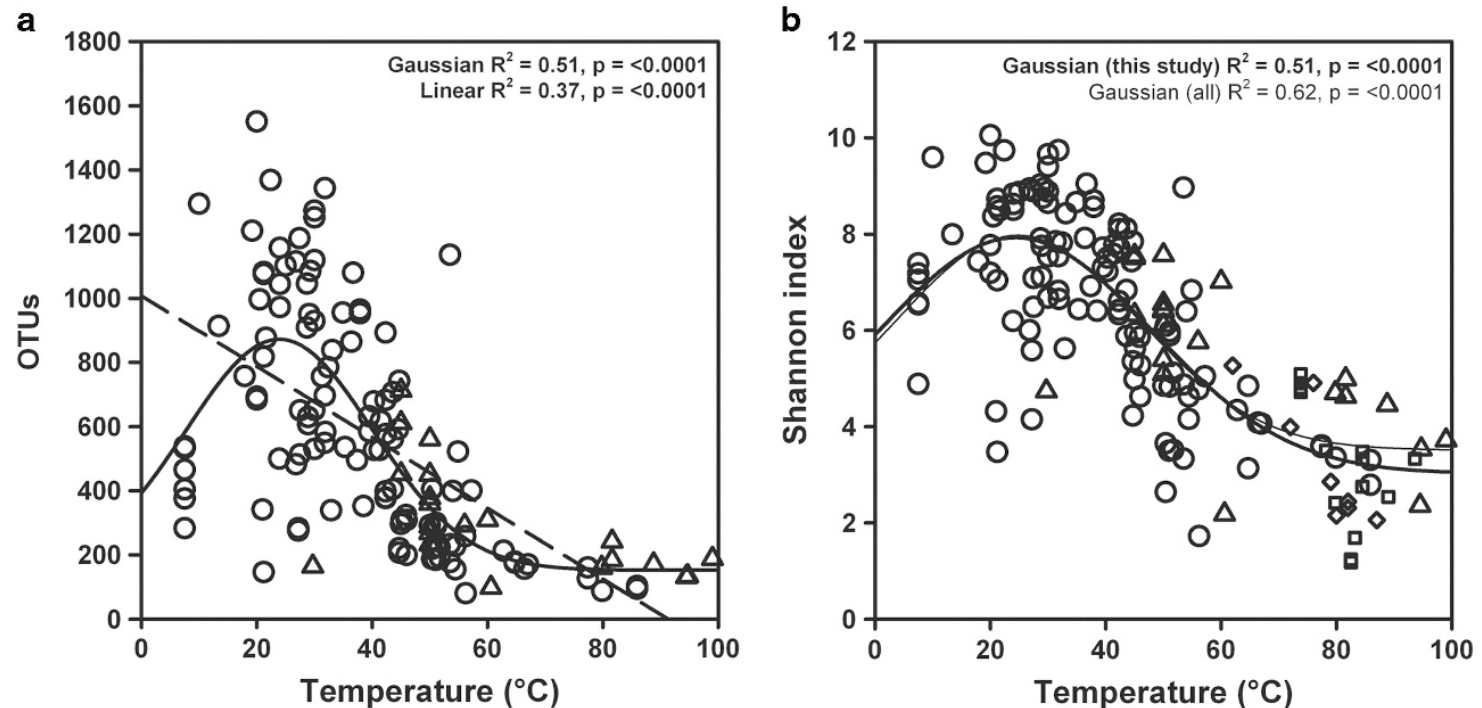

Figure 3 Scatter plots of total OTUs detected (a) and Shannon diversity index (b) versus temperature for neutral-alkaline springs (pH $>5$ ) included in the current study, plus additional sites from previous studies (Cole et al., 2013; Hou et al., 2013). Solid lines represent the best-fit Gaussian four-parameter model to the data. The dashed line represents the best-fit linear model to the data. Diversity indices for our study were calculated using 2730 sequences per spring sample. Symbol shape indicates Canada $(\bigcirc)$, New Zealand $(\Delta)$, China $(\square)$ (Hou et al., 2013) or the United States $(\diamond)$ (Cole et al., 2013).

Hou et al. (2013) amplified the V4-V8 region. Both the studies used Mothur to remove low quality reads and downstream analyses were performed using QIIME. Singletons remained in the analysis, and these studies are therefore compared with our unfiltered data containing singletons. Hou et al. (2013) denoised their data set, whereas Cole et al. (2013) did not, however our analyses suggest that denoising has a minimal effect on the Shannon index (on average a $15.7 \%$ decline; Supplementary Table S2). Alpha diversities for these two studies were rarefied to depths of 978 and 5325, both of which are above the 700-sequence depth at which our samples leveled off. Recognizing the many caveats that these data are not perfectly comparable with ours, they incorporated remarkably well into the relationship, with a high overall $R^{2}$ of 0.62 and peak diversity at $24.4^{\circ} \mathrm{C}$ (Figure 3b).

To obtain a site-independent estimate of the total number of species adapted to different temperature ranges, 16S rRNA gene read sets for each sample were rarefied to 4000 random sequences and pooled into temperature ranges defined to obtain similar numbers of samples in each range. Each temperature range was then rarefied to a depth of 35000 random sequences and OTUs defined at varying clustering levels (Supplementary Figure S5). Temperature ranges were as follows, $<20^{\circ} \mathrm{C}$ (9 samples), $20-30^{\circ} \mathrm{C}$ 
(32 samples), $\quad 30-40{ }^{\circ} \mathrm{C} \quad(26$ samples $), \quad 40-50{ }^{\circ} \mathrm{C}$ (31 samples), $50-60{ }^{\circ} \mathrm{C}$ (30 samples) and $60-100{ }^{\circ} \mathrm{C}$ (20 samples). The highest richness was observed in a temperature range of $20-30{ }^{\circ} \mathrm{C}$, and declined towards more extreme temperatures (Supplementary Figure S5). A similar pattern was observed when clustering OTUs at 97\%, 95\% and 90\% identity, or $97 \%$ with singletons removed from the analysis (Supplementary Figure S5) confirming that the trend is not sensitive to different species definitions, pyrotag sequencing artifacts or the presence of singletons (Neufeld and Mohn, 2005). The $60-100{ }^{\circ} \mathrm{C}$ group had the lowest species richness, despite covering the largest temperature range. This analysis suggests that the decline in diversity with increasing temperature is primarily due to the existence of fewer total taxa adapted to extreme temperatures, rather than to community assembly effects. That is, a high-temperature environment is less diverse than a moderate-temperature environment because the total pool of thermophilic species that may colonize it is smaller, not because a given high-temperature environment is colonized by a smaller fraction of an equally diverse pool of thermophilic species.

Including latitude as a factor along with temperature and $\mathrm{pH}$ in multiple linear regressions showed that latitude accounted for only $7.8 \%$ of the variability in the Shannon index. Plotting Shannon diversity index values versus latitude from the equator for the neutral-alkaline springs resulted in a regression that was not significantly different from zero (Supplementary Figure S6).

\section{Beta diversity patterns}

Nonmetric multidimensional scaling plots were determined based on a matrix of Bray-Curtis community similarities (Figure 2). Acid springs separated from neutral and alkaline springs regardless of temperature. Neutral and alkaline springs separated according to temperature (Figure 2a). An assessment of the relationship between diversity and geographic location (Canada vs New Zealand) also identified a broad separation, although this was primarily due to the relatively larger number of acid springs sampled from New Zealand (Figure 2b). Within the neutralalkaline cluster, samples from the two countries mixed together. Analysis based on a matrix of UniFrac distances showed similar trends to the Bray-Curtis matrix (Supplementary Figure S7).

Shifts in taxon abundances with $\mathrm{pH}$ and temperature Phyla such as the Proteobacteria, Chloroflexi, Firmicutes, Euryarchaeota and Crenarchaeota showed no clear trend in relative abundance across temperature ranges. However, other phyla displayed trends that correlated with temperature. A number of phyla were only observed in samples from below $80^{\circ} \mathrm{C}$, including Cyanobacteria, Acidobacteria, Verrucomicrobia and Planctomycetes. The absence of Cyanobacteria at high temperature is consistent with the established upper temperature limit for photosynthesis $\left(73^{\circ} \mathrm{C}\right.$ ) (Berg, 2011). Crenarchaeota, Deinococcus-Thermus and Aquificae were observed in the highest abundance at $>60{ }^{\circ} \mathrm{C}$. Candidate division OP11 was found in highest abundance at temperatures below $60{ }^{\circ} \mathrm{C}$. Armatimonadetes (OP10) reached its highest relative abundance between $50-80{ }^{\circ} \mathrm{C}$, which is consistent with previous environmental and culture-based studies of this group (Stott et al., 2008; Dunfield et al., 2012) (Supplementary Figure S8a).

$\mathrm{pH}$ was also observed to have some influence on the relative abundances of different phyla, although both Bacteria and Archaea were observed in all pH ranges (Supplementary Figure S8b). Although the relative abundances of phyla such as Proteobacteria, Acidobacteria, Crenarchaeota and Bacteroidetes did not exhibit any clear shifts with $\mathrm{pH}$ across geothermal environments, other phyla such as Planctomycetes, Cyanobacteria, Chloroflexi and Firmicutes increased in abundance above pH 4.5 (Supplementary Figure S8b). Euryarchaeota dominated in low $\mathrm{pH}$ environments $(<4.5)$.

\section{Discussion}

Analysis of over 1.1 million 16S rRNA gene sequence reads from 165 geothermal samples demonstrated broad changes in species richness and community diversity that were consistently related to temperature and $\mathrm{pH}$. Peak diversity for all samples was observed at $\mathrm{pH} 7$ and $24{ }^{\circ} \mathrm{C}$, with decreasing diversity at more extreme conditions. Temperature had the greater influence on OTU richness and Shannon diversity, with $R^{2}$ values of 0.51-0.62 for neutralalkaline springs only (Figure 3), and 0.40-0.44 for all springs (Supplementary Figure S1a and g). A decline in diversity with increasing temperatures above $20^{\circ} \mathrm{C}$ was evident, and although the number of samples from low temperature sites $\left(<20^{\circ} \mathrm{C}\right)$ was limited, the data suggest that lower temperatures also had a negative effect on microbial diversity.

Although not as strong a factor, $\mathrm{pH}$ accounted for $R^{2}$ values between $0.13-0.20$ of the observed variation in diversity indices (Figure 1, Supplementary Figure S1). With all metrics, peak diversity was observed in soils with near neutral pH. Soils with the lowest level of diversity were found in environments with $\mathrm{pH}$ values $<6$ (Figure 1, Supplementary Figure S1). This is consistent with previous work examining the effect of $\mathrm{pH}$ on terrestrial soils (Fierer and Jackson, 2006; Lauber et al., 2009; Chu et al., 2010; Rousk et al., 2010). Above pH 8 it is not clear whether the relationship decreases as $\mathrm{pH}$ increases (forming a bell curve) or if diversity plateaus at near neutral $\mathrm{pH}$. As samples with $\mathrm{pH}>8$ were rare this relationship cannot easily be distinguished.

Our finding of a stronger dependence of alpha diversity on temperature than on $\mathrm{pH}$ contrasts to 
previous studies identifying $\mathrm{pH}$ rather than mean annual temperatures as the strongest influence on microbial species diversity in terrestrial soils (Fierer and Jackson, 2006; Lauber et al., 2009; Chu et al., 2010; Rousk et al., 2010). However, temperatures in soils have a more limited range than geothermal samples (generally $<30^{\circ} \mathrm{C}$ ), and are seasonally highly variable (Nemergut et al., 2005). Arctic soils, for example, are exposed to air temperatures that vary by as much as $75{ }^{\circ} \mathrm{C}$ annually (Environment Canada, 2013). Microbial communities respond rapidly to changes in environmental conditions (Mackelprang et al., 2011) and as growth is expected to occur predominantly in the warm summer months, mean annual temperatures may be a poor indicator of what a soil microbial community is adapted to. Geothermal systems in contrast have more stable temperatures. For example, three geothermal springs in the Canadian Arctic showed annual temperature fluctuations of less than $0.5^{\circ} \mathrm{C}$, despite monthly average air temperatures ranging from -37 to $+5{ }^{\circ} \mathrm{C}$ (Pollard et al., 1999). The lack of a mean annual temperatures-diversity gradient in a sample set including thermally stable and thermally fluctuating soils is therefore not surprising, although studies of more thermally stable ocean waters (Pommier et al., 2007; Fuhrman et al., 2008) and geothermal springs (this study) do show temperature-diversity relationships. However, the relationships we have documented are much stronger than previous temperature-diversity gradients described for oceans, where $R^{2}$ values were only 0.11-0.20 (Pommier et al., 2007; Fuhrman et al., 2008). This may also be related to the relatively narrow temperature range for ocean waters $\left(0-29^{\circ} \mathrm{C}\right)$ (Pommier et al., 2007; Fuhrman et al., 2008). In addition, the use of geothermal samples covering a broad temperature range has allowed us to show for the first time that the relationship of microbial diversity to temperature is not monotonic, but rather a bell curve with peak diversity at about $24^{\circ} \mathrm{C}$.

Previous studies have attempted to correlate diversity with temperature in geothermal sites, with inconsistent results due to several limiting factors as discussed in the Introduction. The lack of consensus is not surprising given the lack of statistical robustness and the possibility for site-specific effects in these studies. They may also have suffered from a focus on samples with temperatures $>55^{\circ} \mathrm{C}$ (Everroad et al., 2012; Cole et al., 2013; Hou et al., 2013) where the relationship is weakest. Decreasing alpha diversity with increasing temperature was most evident in our sample set from $25-65^{\circ} \mathrm{C}$, but above this the relationship flattened out compared with experimental noise (Figure 3, Supplementary Figure S1). It is possible that factors such as chemical composition (for example, sulfide) increase in importance at high temperatures as well (Purcell et al., 2007).

Temperature effects on the species and phylum composition of microbial communities in hot springs have been reported in several studies (Yim et al., 2006; Purcell et al., 2007; Miller et al., 2009; Huang et al., 2011; Everroad et al., 2012; Cole et al., 2013; Hou et al., 2013; Wang et al., 2013). In our study, some phyla showed distinct abundance trends with $\mathrm{pH}$ and temperature, whereas others showed none (Supplementary Figure 8). The dominance of Aquificae, Crenarchaeaota and Deinococcus-Thermus is consistent with previous studies of geothermal sites with temperatures above $50{ }^{\circ} \mathrm{C}$ and neutral pH (Purcell et al., 2007; Tobler and Benning, 2011; Cole et al., 2013; Hou et al., 2013; Wang et al., 2013). The Bacteria and Archaea generally observed in this study have been previously identified in other geothermal springs in Yellowstone National Park (Miller et al., 2009; Cole et al., 2013), Japan (Everroad et al., 2012), Thailand (Purcell et al., 2007) and Tibet (Yim et al., 2006; Huang et al., 2011; Hou et al., 2013; Wang et al., 2013).

The latitude-diversity gradient of von Humboldt is well established for many groups of Eukarya. Owing to the correlation between temperature and latitude, the latitude-diversity gradient is also seen as a temperature-diversity gradient, but it is often attributed causally not directly to temperature but rather to other factors such as NPP (Gaston, 2000; Clarke and Gaston, 2006). In our study a relationship of diversity to temperature was found that was not related to differences in latitude. A regression of the Shannon diversity index versus latitude from the equator was not significantly different from zero for the samples included in this study (Supplementary Figure S6). This verified that sample temperatures were uncoupled from latitude and related trends, and therefore the relationship of diversity to temperature was probably direct. Our study therefore supports a direct effect of temperature on microbial diversity, not a secondary effect via NPP or other factors related to latitude. These findings are consistent with the Metabolic Niche Hypothesis (Clarke and Gaston, 2006) that postulates that there are many energetically viable ways of making a living at optimal temperatures, but that only the most energetically favorable lifestyles can provide an organism with sufficient maintenance energy under extreme temperatures where energy must also be expended for stress tolerance. This hypothesis is particularly appealing for microbes, which display a much broader suite of potential metabolic pathways than do macroflora and macrofauna. The data shown in Supplementary Figure S5 demonstrate that the major factor causing low-diversity communities at high temperature is simply an overall scarcity of species that are adapted to these conditions, rather than a more restricted assembly of an equally diverse pool of species. Oren (2001) applied the same logic to explain the decreasing diversity of microbial communities with increasing osmotic stress. He noted that metabolic pathways like acetogenesis, dissimilatory sulfate reduction and 
autotrophic nitrification were only observed below certain environmental salt concentrations, and proposed that these concentrations represented stress ceilings above which the metabolic processes could no longer provide sufficient energy for maintenance and stress-tolerance mechanisms. The number of viable metabolic lifestyles therefore decreases with increasing stress, translating to a loss of taxonomic diversity. This theorem could equally apply to temperature stress. Besides the requirement for a high energy yield at high stress, a further problem for thermophiles is the thermal instability of certain chemicals. For example, photosynthesis occurs only below $73{ }^{\circ} \mathrm{C}$, in part because of the thermal instability of intermediates of the Calvin-Benson-Bassham Cycle (Berg, 2011). The lack of Cyanobacteria at high temperatures within the current study is consistent with this effect.

There are alternate explanations for our results. One alternative explanation for decreased diversity at high temperature is altered mutation and speciation rates. Thermophiles may have lower mutation rates than mesophiles because they invest more effort into DNA repair (Drake, 2009). NPP rather than temperature per se is also frequently cited as the cause of the latitude-diversity gradient (Clarke and Gaston, 2006). NPP was not measured in this study, however it is unlikely that lithotrophic productivity decreases with temperature (Amend and Shock, 2001).

The data presented here demonstrate that temperature and to a lesser extent $\mathrm{pH}$ are strong drivers of microbial community diversity and richness, accounting together for about half of the total variability in alpha diversity. The relationship is not monotonic but rather a bell curve, with peak OTU richness and Shannon diversity at $24^{\circ} \mathrm{C}$. The data are consistent with theories predicting that low diversity at extreme temperatures is directly related to temperature stress, and that few species are adapted to this stress (Supplementary Figure S5). Although other physical and/or geochemical variables may also be contributing factors (Purcell et al., 2007), temperature alone appears to have a major role in determining microbial diversity.

\section{Conflict of Interest}

The authors declare no conflict of interest.

\section{Acknowledgements}

The work was supported by an Alberta InnovatesTechnology Futures (AITF) New Faculty Award and a Natural Sciences and Engineering Research Council of Canada (NSERC) Discovery Grant to PD, as well as by a Geothermal Resources of New Zealand (GRN) funding to MBS. CES was supported by doctoral fellowships from NSERC and AITF. ALB was supported by a postdoctoral award from NSERC.

\section{References}

Allen AP, Brown JH, Gillooly JF. (2002). Global biodiversity, biochemical kinetics, and the energetic-equivalence rule. Science 297: 1545-1548.

Altschul SF, Gish W, Miller W, Myers EW, Lipman DJ. (1990). Basic local alignment search tool. J Mol Biol 215: $403-410$.

Amend JP, Shock EL. (2001). Energetics of overall metabolic reactions of thermophilic and hyperthermophilic Archaea and Bacteria. FEMS Microbiol Rev 25: $175-243$.

Berg IA. (2011). Ecological aspects of the distribution of different autotrophic $\mathrm{CO}_{2}$ fixation pathways. Appl Environ Microbiol 77: 1925-1936.

Caporaso JG, Bittinger K, Bushman FD, DeSantis TZ, Andersen GL, Knight R. (2010a). PyNAST: a flexible tool for aligning sequences to a template alignment. Bioinformatics 26: 266-267.

Caporaso JG, Kuczynski J, Stombaugh J, Bittinger K, Bushman FD, Costello EK et al. (2010b). QIIME allows analysis of high-throughput community sequencing data. Nat Methods 7: 335-336.

Chao A. (1984). Nonparametric estimation of the number of classes in a population. Scand J Stat 11: 265-270.

Chu H, Fierer N, Lauber CL, Caporaso JG, Knight R, Grogan P. (2010). Soil bacterial diversity in the Arctic is not fundamentally different from that found in other biomes. Environ Microbiol 12: 2998-3006.

Clarke A, Gaston KJ. (2006). Climate, energy and diversity. Proc Biol Sci 273: 2257-2266.

Cole JK, Peacock JP, Dodsworth JA, Williams AJ, Thompson DB, Dong $\mathrm{H}$ et al. (2013). Sediment microbial communities in Great Boiling Spring are controlled by temperature and distinct from water communities. ISME J 7: 718-729.

Currie DJ, Mittelbach GG, Cornell HV, Field R, Guégan JF, Hawkins BA et al. (2004). Predictions and tests of climate-based hypotheses of broad-scale variation in taxonomic richness. Ecol Lett 7: 1121-1134.

Drake JW. (2009). Avoiding dangerous missense: thermophiles display especially low mutation rates. PLoS Genetics 5: e1000520.

Dunfield PF, Tamas I, Lee KC, Morgan XC, McDonald IR, Stott MB. (2012). Electing a candidate: a speculative history of the bacterial phylum OP10. Environ Microbiol 14: 3069-3080.

Edgar RC. (2010). Search and clustering orders of magnitude faster than BLAST. Bioinformatics 26: 2460-2461.

Engelbrektson A, Kunin V, Wrighton KC, Zvenigorodsky N, Chen F, Ochman H et al. (2010). Experimental factors affecting PCR-based estimates of microbial species richness and evenness. ISME J 4: 642-647.

Environment Canada (2013). Canadian climate normals 1971-2000. Available at http://climate.weatheroffice.gc.ca/ climate_normals/results_e.html?stnID=1750andlang =eand $\mathrm{d}$ Code $=1$ andprovince $=$ NUandprovBut=Searchand month1=0andmonth2=12.

Evans KL, Warren PH, Gaston KJ. (2005). Species-energy relationships at the macroecological scale: a review of the mechanisms. Biol Rev Camb Philos Soc 80: 1-25.

Everroad RC, Otaki H, Matsuura K, Haruta S. (2012). Diversification of bacterial community composition along a temperature gradient at a thermal spring. Microbes Environ 27: 374-381.

Faith DP. (1992). Conservation evaluation and phylogenetic diversity. Biol Conserv 61: 1-10. 
Fierer N, Jackson RB. (2006). The diversity and biogeography of soil bacterial communities. Proc Natl Acad Sci USA 103: 626-631.

Forster JR. (1778). Observations Made During a Voyage Round the World, on Physical Geography, Natural History and Ethic Philosophy. G. Robinson: London.

Fuhrman JA, Steele JA, Hewson I, Schwalbach MS, Brown MV, Green JL et al. (2008). A latitudinal diversity gradient in planktonic marine bacteria. Proc Natl Acad Sci USA 105: 7774-7778.

Gaston KJ. (2000). Global patterns in biodiversity. Nature 405: 220-227.

Giggenbach WO. (1994). Variations in the chemical and isotopic composition of fluids discharged from the Taupo Volcanic Zone, New Zealand. I Volcanol Geotherm Res 68: 89-116.

Gilles A, Meglécz E, Pech N, Ferreira S, Malausa T, Martin JF. (2011). Accuracy and quality assessment of 454 GS-FLX Titanium pyrosequencing. BMC Genomics 12: 245.

Grasby SE, Hutcheon I, Krouse HR. (2000). The influence of water-rock interaction on the chemistry of thermal springs in western Canada. Appl Geochem 15: 439-454.

Haas BJ, Gevers D, Earl AM, Feldgarden M, Ward DV, Giannoukos G et al. (2011). Chimeric 16S rRNA sequence formation and detection in Sanger and 454-pyrosequenced PCR amplicons. Genome Res 21: 494-504.

Hawkins BA, Field R, Cornell HV, Currie DJ, Guégan JF, Kaufman DM et al. (2003). Energy, water and broadscale geographic patterns of species richness. Ecology 84: 3105-3117.

Hou W, Wang S, Dong H, Jiang H, Briggs BR, Peacock JP et al. (2013). A comprehensive census of microbial diversity in hot springs of Tengchong, Yunnan Province China using 16S rRNA gene pyrosequencing. PLoS One 8: e53350.

Huang Q, Dong CZ, Dong RM, Jiang H, Wang S, Wang G et al. (2011). Archaeal and bacterial diversity in hot springs on the Tibetan Plateau, China. Extremophiles 15: 549-563.

Humboldt Av. (1808). Ansichten der Natur mit wissenschaftlichen Erläuterungen. J.G. Cotta: Tubingen, Germany.

Knief C, Lipski A, Dunfield PF. (2003). Diversity and activity of methanotrophic bacteria in different upland soils. Appl Environ Microbiol 69: 6703-6714.

Lauber CL, Hamady M, Knight R, Fierer N. (2009). Pyrosequencing-based assessment of soil $\mathrm{pH}$ as a predictor of soil bacterial community structure at the continental scale. Appl Environ Microbiol 75: 5111-5120.

Mackelprang R, Waldrop MP, DeAngelis KM, David MM, Chavarria KL, Blazewicz SJ et al. (2011). Metagenomic analysis of a permafrost microbial community reveals a rapid response to thaw. Nature 480: 368-371.

Miller SR, Strong AL, Jones KL, Ungerer MC. (2009). Bar-coded pyrosequencing reveals shared bacterial community properties along the temperature gradients of two alkaline hot springs in Yellowstone National Park. Appl Environ Microbiol 75: 4565-4572.

Nemergut DR, Costello EK, Meyer AF, Pescador MY, Weintraub MN, Schmidt SK et al. (2005). Structure and function of alpine and arctic soil microbial communities. Res Microbiol 156: 775-784.

Neufeld JD, Mohn WW. (2005). Unexpectedly high bacterial diversity in arctic tundra relative to boreal forest soils, revealed by serial analysis of ribosomal sequence tags. Appl Environ Microbiol 71: 5710-5718.

Oren A. (2001). The bioenergetic basis for the decrease in metabolic diversity at increasing salt concentrations: implications for the functioning of salt lake ecosystems. Hydrobiologia 466: 61-72.

Pennanen T, Liski J, Bååth E, Kitunen VV, Uotila J, Westman CJ et al. (1999). Structure of the microbial communities in coniferous forest soils in relation to site fertility and stand development stage. Microb Ecol 38: 168-179.

Pollard W, Omelon C, Andersen D, McKay C. (1999). Perennial spring occurrence in the Expedition Fiord area of western Axel Heiberg Island, Canadian High Arctic. Can J Earth Sci 36: 105-120.

Pommier T, Canback B, Riemann L, Bostrom KH, Simu K, Lundberg $\mathrm{P}$ et al. (2007). Global patterns of diversity and community structure in marine bacterioplankton. Mol Ecol 16: 867-880.

Price MN, Dehal PS, Arkin AP. (2009). FastTree: computing large minimum evolution trees with profiles instead of a distance matrix. Mol Biol Evol 26: 1641-1650.

Pruesse E, Quast C, Knittel K, Fuchs BM, Ludwig W, Peplies J et al. (2007). SILVA: a comprehensive online resource for quality checked and aligned ribosomal RNA sequence data compatible with ARB. Nucleic Acids Res 35: 7188-7196.

Purcell D, Sompong U, Yim LC, Barraclough TG, Peerapornpisal Y, Pointing SB. (2007). The effects of temperature, $\mathrm{pH}$ and sulphide on the community structure of hyperthermophilic streamers in hot springs of northern Thailand. FEMS Microbiol Ecol 60: $456-466$.

Ramos-Padrón E, Bordenave S, Lin S, Bhaskar IM, Dong X, Sensen CW et al. (2011). Carbon and sulfur cycling by microbial communities in a gypsum-treated oil sands tailings pond. Environ Sci Technol 45: 439-446.

Rousk J, Bååth E, Brookes PC, Lauber CL, Lozupone C, Caporaso JG et al. (2010). Soil bacterial and fungal communities across a pH gradient in an arable soil. ISME J 4: 1340-1351.

Stott MB, Crowe MA, Mountain BW, Smirnova AV, Hou S, Alam $\mathrm{M}$ et al. (2008). Isolation of novel bacteria, including a candidate division, from geothermal soils in New Zealand. Environ Microbiol 10: 2030-2041.

Takai K, Nakamura K, Toki T, Tsunogai U, Miyazaki M, Miyazaki J et al. (2008). Cell proliferation at $122^{\circ} \mathrm{C}$ and isotopically heavy $\mathrm{CH}_{4}$ production by a hyperthermophilic methanogen under high-pressure cultivation. Proc Natl Acad Sci USA 105: 10949-10954.

Tobler D, Benning L. (2011). Bacterial diversity in five Icelandic geothermal waters: temperature and sinter growth rate effects. Extremophiles 15: 473-485.

Wang S, Hou W, Dong H, Jiang H, Huang L, Wu G et al. (2013). Control of temperature on microbial community structure in hot springs of the Tibetan plateau. PLoS One 8: e62901.

Yim LC, Hongmei J, Aitchison JC, Pointing SB. (2006). Highly diverse community structure in a remote central Tibetan geothermal spring does not display monotonic variation to thermal stress. FEMS Microbiol Ecol 57: 80-91.

Supplementary Information accompanies this paper on The ISME Journal website (http://www.nature.com/ismej) 